\title{
Enquiry, Engagement, and eLearning: Three Perspectives on a Student-Centred, Online, Enquiry-Based Course
}

\author{
Jacqueline Murray, Natalie Giesbrecht, \& Samuel Mosonyi \\ University of Guelph
}

In the 2011 Winter semester, the University of Guelph engaged in a pedagogical experiment: an online first-year seminar. This article is a conversation about the learning journey that surrounds this seminar, as experienced by three participants: Jacqueline Murray (JM), Professor of History and Director of the FirstYear Seminar Program (FYS); Natalie Giesbrecht (NG), Manager, Distance Education and a Distance Learning Specialist; and Samuel Mosonyi (SM), an undergraduate student who was enrolled in the course. We reflect upon the online seminar and discuss the technology and pedagogy, student learning experience, and process of online interaction. We conclude that this seminar, an innovation in both enquiry-based learning and first-year seminars, is arguably comparable with classroom-based offerings.

\section{Introduction}

$\int$

M: I have been facilitating first-year seminars since 2004. The First-Year Seminar (FYS) Program provides small, intensive, interdisciplinary learning experiences for first-year students. Over the years, I have developed seminars on a variety of interdisciplinary themes. Consequently, when the opportunity to develop a seminar on The Politics, Science, and Culture of Hunger arose, I was most enthusiastic. This seminar would be offered online, something that had never occurred in the FYS Program, which generally prided itself on close faculty-student contact.

First-year seminars are capped at eighteen students to ensure interactivity and engagement. When I facilitate a face-to-face group, I employ a specific pedagogy: closedloop, reiterative, enquiry-based learning (EBL) (Barrows, 1986; Murray \& Summerlee, 2007; Summerlee \& Murray, 2010). Enquiry-based learning is a pedagogy that is both engaging and challenging. It fosters deep learning and transferable skills and so meets the learning objectives of the FYS Program.

Enquiry-based learning is part of the pedagogical constellation that includes problem-based learning (PBL) and inquiry-based learning (IBL). PBL is less a specific pedagogy than an umbrella term that includes multiple approaches to learning that focus on a problem. Enquiry and inquiry-based learning focus on the process of research 
and investigation. The inquiry approach at McMaster University looks to "new knowledge and new understanding" (Hudspith \& Jenkins, 2001). Enquiry-based learning is more focused on the process of questioning, using cases to encourage open-ended enquiry rather than to arrive at solutions (Summerlee \& Murray, 2010).

I was committed to using EBL in the online seminar, given my experience with the pedagogy. Admittedly, having had no previous experience with online learning, I had no clear idea what this might actually entail.

Enquiry-based learning is a more process-oriented than content-oriented pedagogy. Students analyze a number of scenarios pertaining to the seminar's theme (Garrison \& Cleveland-Innes, 2005). These examples provide the context for student learning. Students develop their analytical, research, and presentation skills, and engage in deep learning as they analyze complex multidisciplinary issues and consult the research necessary in order to understand them.

I developed a number of cases that examine the complexities of hunger in both global and North American contexts, for use in the online seminar. Through these examples, students would confront issues of food security in refugee camps, genetically modified foods that could help alleviate hunger, competing or contradictory government policies, and strategies to address domestic hunger. I developed the theme of the seminar and its scenarios in the same way as I would for a face-to-face seminar, but I had no idea how the course could be structured for online delivery or how the cases would work in an online environment.

\section{Course Design and Experience}

NG: At the University of Guelph, most online courses are asynchronous to provide students with scheduling flexibility. The seminar followed this delivery model, despite the innovative pedagogy. It differed from other online offerings by providing an intensive, small group learning experience, and it was the first to use EBL pedagogy as a framework. Thus, it was apparent from the beginning that a creative and well thought-out course design would be crucial for student success, and that the course development would be a learning journey unto itself (Lock, 2002). As a starting point, the design needed to encourage collaboration. To facilitate this, the students were divided into smaller learning groups of six that worked together throughout the semester.

SM: For the students in the seminar course, EBL was a new experience, having been used to the standard lecture, essay, and exam courses. On the course website, EBL was thoroughly defined and easy to understand. A scenario was provided with certain facts, which we then analyzed according to what we knew, and what we did not know. Then, we identified learning issues, created research questions, and distributed them amongst ourselves.

JM: Enquiry-based learning is a student-centred and student-driven pedagogy. The facilitator does not lecture, and does not provide factual background or theoretical grounding. Students only receive an outline about how to proceed, by means of working through a brief sample case in the first class. Then, they jump into their first full case analysis. The challenge for us was discovering how to provide the same kind of information and introductory experience for an online seminar (Hiltz, 1998).

NG: As Jacqueline and I began to develop the course, we realized we needed to fully understand each other's contexts, perspectives, and goals. When I begin to work with a faculty member, we discuss the instructional design process and the best practices of online course development. We work to develop course objectives, activities, and assessments that are aligned and provide the solid foundation for a successful course. As with any course development, Jacqueline and I worked through these processes and practices.

JM: Enquiry-based learning does not fit conventional course design, either classroom-based or online; the seminars are not ordered by units of content or a set number of lectures and labs. The challenge, in this case, was how best to organize and present the course information. The course structure also needed to reinforce the non-hierarchical role of the facilitator. In EBL, the facilitator functions as an integrated member of each learning group rather than an authoritative figure driving the learning.

NG: The goal was to create a course design that would foster student-driven learning. The challenge, however, was to build it so that it stayed true to the EBL model, while acknowledging that the inclass experience cannot be mirrored online.

\section{Media and Learning Process}

JM: In order for Natalie to decide how to structure the 
course online, she needed to understand EBL in depth. We began with a description of the process, what the students do, and what the learning objective is for each step in the process. Words seemed to complicate rather than clarify, so I endeavoured to map out the process visually, on chart paper, hoping to convey the dynamic and integrated nature of EBL.

NG: The chart opened a dialogue between Jacqueline and I and resulted in a hand-drawn diagram that evolved into a multimedia object (Figure 1). This then became a key teaching tool to communicate to the students how EBL works, taking students through each of the steps in a clear and concise manner.

SM: The media object helped to introduce students to the EBL process. The most beneficial demonstration for me, however, was a video of a faceto-face EBL case analysis. The video shows a group of students who were presented with a case and analyzed it, demonstrating how EBL works. The video helped us understand that this course was going to be quite different from any other. We would be required to question our assumptions, even our most intrinsic values.

JM: The goal of the video was to model how to approach a case and analyze it. It also demonstrated the interaction and discussion that occurs in an EBL

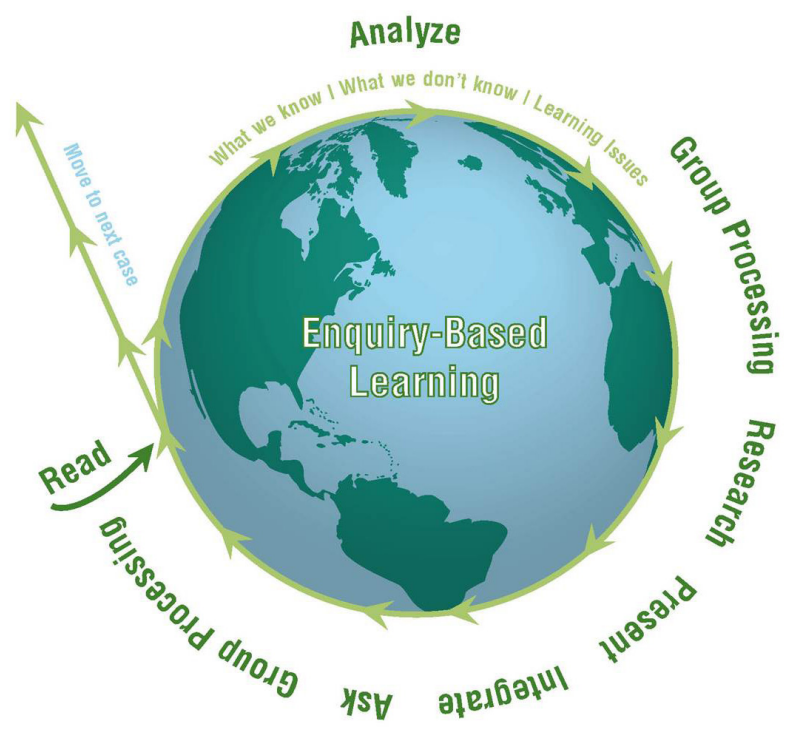

Figure 1

Enquiry-Based Learning Media Object

(Copyright 2010 by N. Giesbrecht and J. Murray) group. The video shows the tentativeness, bewilderment, excitement, and intellectual joy that comes with analyzing an EBL case. Consequently, I was pleased that the online students appreciated videos and were able to use them as a guide for their virtual learning groups.

NG: The video was intended to be a starting point for students to become acquainted with the EBL process. Even so, I was concerned that the students might not translate what they saw in the in-class video into their online, asynchronous seminar. A key issue then was how to create an online environment that would appear familiar from the video as well as take advantage of the available technical tools without creating additional barriers for students (Brown, 1997). This sparked a discussion about appropriate educational technologies to support the learning.

\section{Educational Technology}

NG: We needed to consider that most first-year students would be unfamiliar with online learning. Both the learning environment and EBL process would be new, so it was important to develop explicit instructions and select tools that were easy to use.

The tools became the technological framework that supported the pedagogy and encouraged the highlevel interaction required of EBL. We provided two key spaces in which students would interact. The first was the discussion forums in Desire2Learn (D2L), the University of Guelph's learning management system. These spaces afforded the students the ability to conduct asynchronous discussion of the cases but only allowed for linear interaction and were not sufficiently flexible for collaborative work.

To allow for greater immediacy and spontaneity - and a certain degree of synchronous interaction - the second tool we selected was a third party wiki called Wikispaces. This was a private workspace, visible only to the specific learning group. The wiki pages mirrored the key steps of the EBL process: Analyze, Present Research, and Integrate Research into the scenario (Figure 2). These pages worked in conjunction with the discussion forums. Depending on the step in the process, students moved back and forth between the discussion forums and the wiki.

SM: Initially, we weren't familiar with all of the technologies used for the seminar, and were nervous about this challenge. However, gradually, we became 


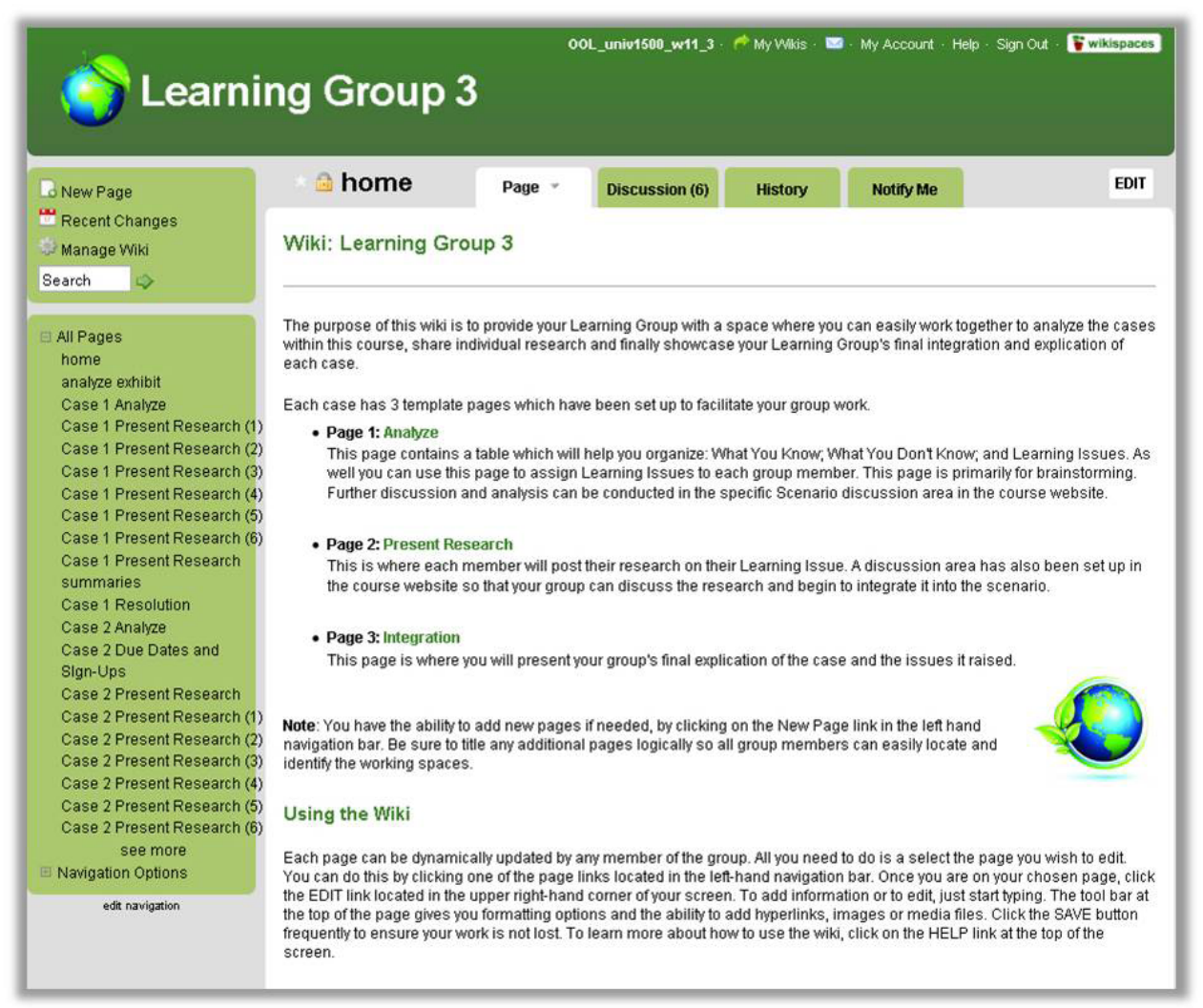

Figure 2

Wiki Set-Up: The Politics, Science, and Culture of Hunger

comfortable with the technology and with accessing the learning environments.

The wiki was our main presentation tool. Initially, our presentations were text-based, but as the semester progressed we became comfortable enough to embed pictures, videos, and other media. For example, in a case that required us to analyze why a supply convoy was delayed, one presentation used satellite imaging to analyze the terrain, while another included an infographic of such quality that it seemed professional.

Our learning group also used the wiki to reach consensus. From the start, we created rules and a voting procedure so that we had an efficient and democratic way to make decisions. We also created separate wiki pages for each group member so that we had an individual space to present research. Another page was designated for the analysis and synthesis of our collective work. The wiki provided a flexible framework and gave each group creative capacity to develop it in a context-appropriate manner (Lock, 2002).

NG: Groups became so cohesive and integrated that they began to finish each other's thoughts and enrich each other's contributions. Through the use of individual colours, the integration and conversational flow was visible on the wikis, as students completed each other's sentences and expanded upon each other's ideas (Figure 3).

\section{Discussion and Interaction}

JM: An important consideration for both group dynamics and the learning environment was that each learning group worked independently. The students were vaguely aware that there were multiple groups, but these did not interact or see each other's work. Rather, each group had its own forums in D2L and its own wiki, so each group was free to express itself without concern that strangers might have access. This meant that the students were uninhibited in their brainstorming and could try out ideas, no matter how wild they might seem at first glance. One concern about experimenting with online learning was the uncertainty of whether the students would be able 


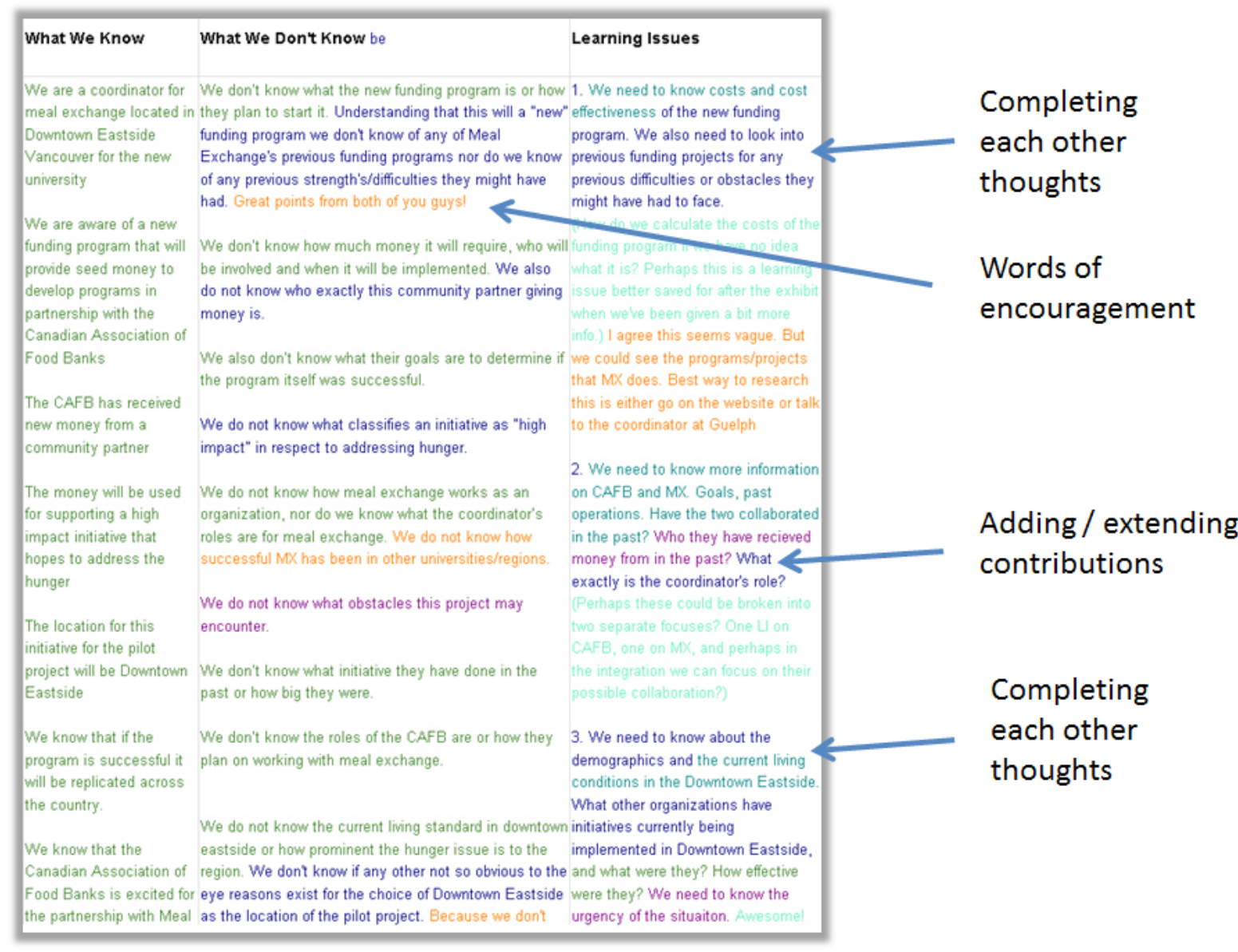

Figure 3

Student Interaction

to get to know one another. How could we provide the equivalent of those five minutes of chatting that happen while waiting for class or packing up afterwards? These are the times when so many social bonds are formed.

NG: We created another social environment with a forum dedicated to introductions, a fairly standard practice in online courses to create rapport and establish community (Palloff \& Pratt, 1999). The introductions provided a starting point, not only for the students to develop socially but also for the instructor to develop a social presence.

JM: I wanted my introduction to model an open yet relevant profile of myself, to convey a tone of warmth, while also conveying to the students that, like them, I consider hunger and social justice to be important. But the mood also needed to be light enough to avoid discouraging students from taking a chance on this new course. Students were asked, "What do you hunger for?" which allowed them to contextualize their introductions in various ways and reveal their personalities. The strategy worked well. They revealed what inspired them and how they wanted to make a difference in the world.

SM: The introductions helped to develop familiarity among group members and revealed everyone's diverse values and perspectives. We gradually understood and respected these differences in perspective that often led to vigorous debate (Jonassen, Peck, \& Wilson, 1999). Aside from the introductions, there were a number of social spaces incorporated into D2L for casual interactions. A forum called Cool Stuff was a place for group members to post articles or videos relevant to the seminar, or notices about lectures and events on campus. The Coffee Shop was an informal space for chat about anything unrelated to the seminar. Within these spaces we offered each other encouragement and support (Garrison, 2007).

NG: The introductions were a starting point 
that led to higher levels of engagement. As group rapport developed, some groups began to engage in almost synchronous conversation in the asynchronous discussion forums. Time stamps for each post reveal that students were posting just minutes apart.

JM: In the classroom, students work together in intensely engaging ways and know each other's strengths and weaknesses. Because facades dissolve quickly, groups can develop true community. It was not clear if a similar depth of relationship could develop asynchronously, without the usual touchstones of expression, gestures, and laughter. This concern proved unfounded. Individuals shone through and genuine relationships developed. Each group had a distinct personality, unique group dynamics, and approaches to problem solving.

SM: The various virtual spaces - including the discussion forums, the wiki, and the informal chat rooms - helped us envision each other as fellow students. It helped us to put thoughts and personalities to names. This enhanced each member's commitment to the group, just as in a face-to-face group. As these relationships deepened, there was increasing engagement with the seminar by all of the group members.

\section{Reflections}

JM: The students in The Politics, Science and Culture of Hunger embraced the multidisciplinary perspectives necessary to understand such a complex issue. The ability to work together with students from various disciplines helped everyone to open their eyes to the array of approaches necessary to understand any issue.

NG: Jacqueline and I devoted considerable time to designing the seminar and selecting the educational technologies. We were ambitious from the beginning but were conscious not to overburden the students with complicated tools and steep learning curves. From the perspective of an instructional designer, this course provided an opportunity to be creative and push the boundaries beyond those of the typical online, contentbased, modular course.

JM: Natalie and I worked together very closely and enthusiastically, meeting once or twice a week in the semester leading up to the course launch and continuing to meet while it was offered. This allowed us to make small adjustments but also reflects the close collaborative relationship we developed and a sense of joint ownership we felt for what we had created. We each learned from the other: I am no techno-wizard and had never taught online, so I developed a deeper understanding of learning technology and the innovative potential of online learning.

SM: One of the most beneficial aspects of the seminar was the process-oriented approach that focused on skill development as opposed to content. With EBL, the faculty facilitator is a member of the group, and the students develop skills by taking ownership of their learning. Because the facilitator does not act as an authority, students have considerable room to let their skills shine. As members of a learning community, we depended on each other to participate and collaborate to achieve our learning outcomes (Palloff \& Pratt, 1999).

JM: The seminar was highly successful in terms of learning outcomes. The students were engaged, developing skills of analysis, research, and integration. We expect longitudinal research, after a number of iterations of the seminar, will provide more information on the effectiveness of student learning and student experience within the online context. Based on my own years of experience with face-to-face EBL, I would judge the online seminar to be of equal quality.

\section{Conclusions}

SM: The class was interesting and unique for me personally. As a first-year student, I had only experienced large lectures; this seminar embodied a whole new style of learning. The trek into the unknown was a rewarding experience for me and the other group members; we learned how to work effectively as a team and were all pushed outside of our safe zones. Our leadership skills were also bolstered, as each group member took on the role of leader at some point in the case analysis. The process-oriented approach allowed us to develop critical, analytical, research, and presentation skills.

NG: We - faculty member and instructional designer - began this course development project with different but complementary expertise, though we did not realize this at first. We worked closely to integrate our two perspectives in order to develop an innovative online course that combines the learner-centred approach of EBL with the best practices of online learning. Ultimately, it was a collegial and collaborative relationship that led us to develop a successful course. This, in turn, had a great impact on the student experience. 
JM: This seminar was a learning journey for all of us: faculty, instructional designer, and students. Creating this seminar paralleled students' experience of it: it involved teamwork and required the ability to view issues from multiple perspectives, to accommodate different perspectives, and to respect different expertise. It was an example of enquiry-based learning at its best, flourishing in an asynchronous, online environment.

\section{References}

Barrows, H. S. (1986). A taxonomy of problem-based learning methods. Medical Education, 20, 481486.

Brown, A. (1997). Designing for learning: What are the essential features of an effective online course? Australian Journal of Educational Technology, 13(2), 115-126.

Garrison, D.R. \& Cleveland-Innes, M. (2005). Facilitating cognitive presence in online learning: Interaction is not enough. American Journal of Distance Education, 19(2), 133-148.

Garrison, D.R. (2007). Online community of inquiry review: Social, cognitive, and teaching presence issues. Journal of Asynchronous Learning Networks, 11(1), 61-72.

Hiltz, S.R. (1998). Collaborative learning in asynchronous learning networks: Building learning communities. (Report No. 019 256). Orlando, FL. Retrieved from ERIC database.

Hudspith, B. \& Jenkins, H. (2001). Teaching the art of inquiry. Halifax, NS: Society for Teaching and Learning in Higher Education.

Jonassen, D.H., Peck, K., \& Wilson, B. G. (1999). Learning with technology: A constructivist approach. Upper Saddle River, NJ: Prentice-Hall.

Lock, J.V. (2002). Laying the groundwork for the development of learning communities within online courses. Quarterly Review of Distance Education, 3(4), 395-408.
Murray, J \& Summerlee, A.J.S. (2007). The impact of problem-based learning in an interdisciplinary first-year program on student learning behaviour. Canadian Journal of Higher Education, 37(3), 85-105.

Palloff, R.M. \& Pratt, K. (1999). Building learning communities in cyberspace. Effective strategies for the online classroom. San Francisco, CA: Jossey-Bass.

Summerlee, A. \& Murray, J. (2010). A study of the impact of enquiry-based learning on academic performance and student engagement. Canadian Journal of Higher Education, 40(2), 78-94.

\section{Biographies}

Jacqueline Murray is Professor of History and Director of the First-Year Seminar Program at the University of Guelph. She is an advocate of enquiry-based learning and engages in research on its learning outcomes and student experience.

Natalie Giesbrecht is Manager, Distance Education and a Distance Learning Specialist in the Centre for Open Learning and Educational Support at the University of Guelph.

Samuel Mosonyi is an undergraduate student at the University of Guelph. He is pursuing a double major in Political Science and Criminal Justice and Public Policy. 